\title{
On the Use of Programmable Metasurfaces in Vehicular Networks
}

\author{
Taqwa Saeed, Waqar Aziz, Andreas Pitsillides, Vasos Vassiliou, Ian Akyildiz Hamidreza Taghvaee, Sergi Abadal \\ Dept. of Computer Science \\ University of Cyprus \\ tkhair01, aziz.waqar-ali, Andreas. Pitsillides, vasosv, ian.Akyiliz@cs.ucy.ac.cy \\ Dept. of Computer Architecture \\ Universitat Politècnica de Catalunya \\ taghvaee, abadal@ac.upc.edu \\ Christos Liaskos, Ageliki Tsioliaridou \\ University of Ioannina CSE \& \\ FORTH Greece \\ cliaskos@uoi.gr, atsiolia@ics.forth.gr \\ Sotiris Ioannidis \\ School of Computer Engineering \\ Technical University of Chania \\ sotiris@ece.tuc.gr \\ Ehizogie Emoyon-iredia, Marios Lestas \\ Dept. of Electrical Engineering \\ Frederick University Cyprus \\ st015229,eng.lm@frederick.ac.cy
}

\begin{abstract}
Metasurface-based intelligent reflecting surfaces constitute a revolutionary technology which can serve the purpose of alleviating the blockage problem in mmwave communication systems. In this work, we consider the hypersurface paradigm complementing the software defined metasurface with an embedded controller network in order to facilitate the dissemination of reconfiguration directives to unit cell controllers. For the first time, we describe the methodology with which to characterize the workload within this embedded network in the case of the metasurface tracking multiple users and we use a vehicular communications setting to showcase the methodology. Beyond that, we demonstrate use cases of the workload analysis. We show how the workload characterization can guide the design of information dissemination schemes achieving significant reduction in the network traffic. Moreover, we show how the workload, as a measure of the consumed power, can be used in designing energy efficient communication protocols through a multi-objective optimization problem maximizing the achieved utilization while at the same time minimizing the workload incurred.
\end{abstract}

\section{INTRODUCTION}

Existing wireless communication technologies, as for example dedicated short range communication (DSRC) and long-term evolution (LTE) in vehicular communications have been shown to pose limitations on the achievable speeds, and mmwave and Thz communications are currently being considered to overcome the bandwidth scarcity problem of next generation capacity demanding networks [1]. However, mmwave wave signals are highly dependent on directional MIMO antenna technologies which employ advanced beamforming techniques to achieve the desired directionality and receiver alignment and are thus challenged in high device mobility cases, e.g., vehicles [2]. Moreover, mmwave communication is susceptible to blockage leading to non-line of sight (NLOS) channels which significantly degrade performance [3]. A revolutionary technology which can counter for this problem is intelligent reflective surfaces (IRS) which can offer virtual line of sight paths in NLOS scenarios [4].

A key enabling technology for such IRS is metasurfaces (MSs) which comprise of repeated subwavelength unit cells in 2D structures, referred to as the meta-atoms, offering precise control over the impinging electromagnetic waves thus outperforming competing technologies as for example phased arrays [5]. Recent literature exhibits a significant interest in the design and analysis of IRS based systems unravelling their fundamental properties and limitations [6]. A number of efforts have also been reported towards the hardware implementation of this technology. Their consideration, however, in vehicular communication systems has so far been limited and this work is motivated by this gap [7]. Moreover, previous work on the use of MSs in wireless communications has been largely based on the assumption that each of the unit cells acts as an independent phase/amplitude shifter [8]. However, such an approach does not consider the MS coding procedure and the way with which reconfiguration directives are disseminated to the unit cells and may not be realizable in practice as the couplings between adjacent elements are neglected.

In this work, we adopt a different approach, taking into consideration the hardware implementation, the MS coding required to realize beam steering out of a set of prespecified functionalities [9], verified using extensive CST simulations and the methodology with which messages are disseminated to the controllers. We build upon the Hypersurface architecture complementing the Software Defined MS with an embedded network of controllers for the dissemination of the reconfiguration messages to the unit cells [10]. Such a controller network solves the scalability problems of direct wiring from the input gateway to each of the unit cells.

Based on this architecture, we explore the potential use of MSs to serve V2X communications and characterize the performance in terms of the workload incurred and the capacity achieved. In our previous work, we have characterized the workload in the case of the MS tracking a single user [11]. Here, we extend the approach to the case of two users and characterize the workload in the case of the MS assisting communications between vehicles. Realistic mobility traces extracted from the VISSIM simulator are considered [12]. The basic scenario, as depicted in Fig. 1, involves a stationary vehicle close to an intersection, utilizing a MS installed at 


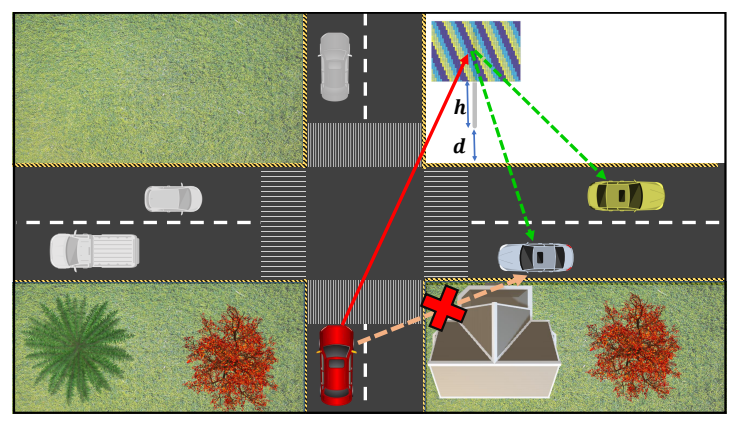

Fig. 1: System scenario.

the intersection to communicate information to other moving vehicles which reside on the lane perpendicular to the one on which the stationary vehicle resides.

In the first scenario, we assume tracking of a single vehicle as it moves. In the second case, we consider tracking of 2 vehicles moving in opposite directions, aiming to explore if and by how much the workload may increase when communication is established with 2 vehicles instead of one. Further, we explore two use cases of the workload analysis. First, we take advantage of the spatio-temporal characteristics of the workload in the case of linear movements of the receivers, which is typical in vehicular environments. The goal is to quantify the degree with which traffic can be reduced within the embedded controller network, when multi-casting is used to convey identical reconfiguration signals along controllers residing in the same row. Secondly, we employ a simple pathloss model for the path between the MS and the receiver, to show how the workload analysis can be used as a baseline to optimize MS-assisted message dissemination in an energy efficient manner. We consider the scenario of switching between users in order to serve them sequentially and explore the problem of maximizing the overall utility in terms of the offered aggregate sending rate subject to the simultaneous objective of minimizing the workload and thus the energy consumption within the MS. Our results indicate an optimal switching period of $300 \mathrm{~ms}$. It is important to note here that although energy efficiency for MSs has been addressed in literature, e.g., [13], here we specifically address optimizing energy consumption in the embedded controller network which is a feature limited to HyperSurfaces.

The paper is organized as follows. In Sec. II the MS coding is presented and the workload incurred by the tracking of multiple users is discussed. Sec. III demonstrates the reduction in the incurred workload when multicasting is employed within the controller network. In Sec. IV the relationship between the workload and the system utility is investigated. Finally, the paper is concluded in Sec. V.

\section{Multi-User WorKLOAD}

A significant aspect of the MS operation is the "workload". The latter terminology refers to the number of reconfigurations that need to be applied to each of the unit cells to realize a particular functionality. Why is the workload important? Based on the Hypersurface architecture [10], packetized reconfiguration directives must be disseminated from the input Gateway
(GW) to each controller via the embedded controller network with each hop in this dissemination procedure associated with a specific power consumption and communication delay. Since power consumption and reconfiguration delay optimization is critical in MS operation, efficient workload management and optimization should be taken into account in different aspects of the MS protocol design.

Below, we describe the procedure with which the workload is obtained for the case of beam splitting on the MS such that the impinging wave is split into two components. The first step takes the form of determining a function $f($.$) whose inputs$ are the desired reflection angles $r_{1}$ and $r_{2}$ of the reflected waves and its output is the vector of $N$ controller states $c$ realizing the beam splitting with the aforementioned reflection angles, such $c=f\left(r_{1}, r_{2}\right)$. Each of the elements of the vector $c$ assumes one of $L$ states. The angle $r_{i}, i \epsilon\{1,2\}$ is a two dimensional vector comprising of the azimuth angle $\phi_{i}$ and the elevation angle $\theta_{i}$ such that $r_{i}=\left[\phi_{i}, \theta_{i}\right]^{T}$. The function $f($.$) is determined according to the procedure described in the$ MS Coding Subsection later on.

The second step involves determining the number of messages $m$ that need to be sent to the controller states to facilitate a reflection angle change from $\left(r_{1}(k), r_{2}(k)\right)$ to $\left(r_{1}(k+1)\right.$, $\left.r_{2}(k+1)\right)$. This is calculated by counting the number of the corresponding elements of $c(k)=f\left(r_{1}(k), r_{2}(k)\right)$ and $c(k+1)=f\left(r_{1}(k+1), r_{2}(k+1)\right)$ which report a change in their state. This will dictate the function $g($.$) such that$ $m=g\left(r_{1}(k), r_{2}(k), r_{1}(k+1), r_{2}(k+1)\right)$. The function $g($.$) can be expressed analytically as m=h^{t} h$ where $h=$ $\operatorname{sgn}(c(k+1)-c(k))$ with $\operatorname{sgn}(x)$ representing the signum function. As two receivers move along their trajectories, this will induce time profiles of desired reflection angles $r_{1}(t)$ and $r_{2}(t)$. As the MS cannot reconfigure continuously (the reconfiguration procedure is not instantaneous) a reconfiguration policy is adopted which dictates the reconfiguration instants.

Several reconfiguration policies may be adopted as for example periodic reconfigurations with period $T$, often encountered in feedback-based applications, or event-driven approaches where the reconfiguration occurs when a particular condition is met. Previous work [11] has considered reconfigurations every angular step $\alpha$ i.e. when either the azimuth or elevation angle changes by $\alpha^{\circ}$ relative to the previous reconfiguration. This is relevant to the case that reconfigurations occur every time the receiver exits the beam width, reasonable in the sense that the received signal strength drops significantly upon such an exit.

In the case of multiple receivers, the condition becomes that a reconfiguration is realized upon either the azimuth or the elevation angle of either user changes by $\alpha^{\circ}$ relative to the previous reconfiguration. We may formalize the above arguments as follows: Let $t_{k}, k=0,1, .$. denote the time instants at which reconfigurations occur with the corresponding sequence of MS states and reflection angles denoted by $c(k)$ and $r_{i}(k)$ $i \epsilon\{1,2\}$, respectively. The sequence $t_{k}$ may be generated by defining $t_{k+1}$ when $t_{k}$ is known. We define as $t_{\theta_{i}(k+1)}=$ $\arg \min _{t>k}\left\|\theta_{i}(t)-\theta_{i}(k)\right\| \geq \alpha$. Similarly we define $t_{\phi_{i}(k+1)}$. 
Then $t_{k+1}=\min _{i}\left(t_{\theta_{i}(k+1)}, t_{\phi_{i}(k+1)}\right)$. Having defined the sequence of controller states $c(k)$, quantities of interest include the sequence of the number of messages that need to be sent by the input GW $m(k)=g\left(r_{1}(k-1), r_{2}(k-1), r_{1}(k), r_{2}(k)\right)$, the total number of messages $M=\sum_{k \epsilon K} m(k)$, and the number of reconfigurations $m_{i}$ of each controller $c_{i}$ evaluated as $m_{i}=\sum_{k=1}^{K} \operatorname{sgn}^{2}\left(c_{i}(k)-c_{i}(k-1)\right)$

\section{A. Metasurface Coding}

The direction of reflection can be engineered by an appropriate linear phase gradient [14], [15]. Assuming that the MS imposes the phase profile $\Phi(x, y)$, we assign the virtual wave vector $\mathbf{k}_{\Phi}=\nabla \Phi=\partial_{x} \Phi \hat{x}+\partial_{y} \Phi \hat{y}$ ( $\partial_{x}$ and $\partial_{y}$ denote partial derivatives). The momentum conservation law can be expressed as

$$
\begin{aligned}
& k \sin \theta_{i} \cos \varphi_{i}+\partial_{x} \Phi=k \sin \theta_{r} \cos \varphi_{r}, \\
& k \sin \theta_{i} \sin \varphi_{i}+\partial_{y} \Phi=k \sin \theta_{r} \sin \varphi_{r},
\end{aligned}
$$

where $\partial_{x} \Phi$ and $\partial_{y} \Phi$ describe the imposed phase gradients in the $x$ and $y$ directions, respectively, and the subscripts $i$ and $r$ denote incident and reflected (scattered) waves, respectively. Assuming air as the host medium the required phase reads

$$
\Phi_{m n}=\frac{2 \pi D_{u}\left(m \cos \varphi_{r} \sin \theta_{r}+n \sin \varphi_{r} \sin \theta_{r}\right)}{\lambda_{0}}
$$

The required E-field distribution of multi-beam can be obtained by superposing the E-field of each beam. Assume that we have $K$ users, the superposition E-field of the MS is expressed as follows:

$$
E(\theta, \phi)=\sum_{n=1}^{N} \sum_{m=1}^{M} e^{j k_{0} \zeta_{m n}} \sum_{k=1}^{K} e^{j \Phi_{m n}\left(\theta_{r k}, \phi_{r k}\right)}
$$

Finally, $\zeta_{m n}(\theta, \varphi)$ is the relative phase shift of the unit cells with respect to the radiation pattern coordinates, given by

$$
\zeta_{m n}(\theta, \varphi)=D_{u} \sin \theta\left[\left(m-\frac{1}{2}\right) \cos \varphi+\left(n-\frac{1}{2}\right) \sin \varphi\right]
$$

\section{B. Simulation Scenario}

The basic scenario as depicted in Fig. 1 involves a stationary vehicle close to a signalized intersection, utilizing a MS installed on the intersection to communicate information to other moving vehicles which reside on the lane perpendicular to the one that the stationary vehicle resides. The communicating vehicles do not share a line of sight (LOS) due to blockage from vehicles and buildings and thus utilize the MS in order to offer virtual LOS via reflection. The transmitting vehicle is assumed stationary to decouple its mobility from the mobility of the receiving vehicle. Such a scenario may represent a situation where an unexpected event such as an accident blocks the movement of the transmitting vehicle which then aims to send critical data, as for example images or video to vehicles in the vicinity for the purpose of cooperative driving or safety applications.

The vehicle traces are generated using the VISSIM software to render the adopted mobility model realistic. Penetration rates of $100 \mathrm{veh} /$ hour are considered with an average speed of

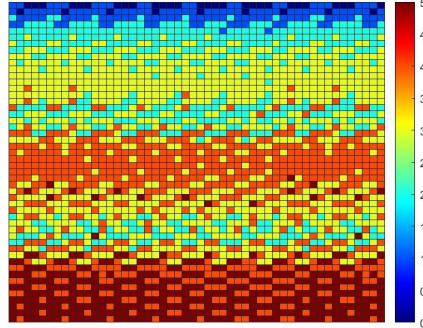

(a) Single User

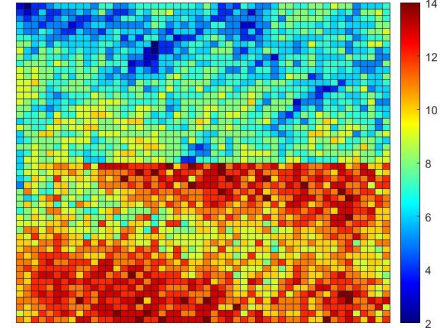

(b) Multi User with Row Sectoring
Fig. 2: Reconfiguration Frequency in each of the controllers.

$30 \mathrm{~km} / \mathrm{h}$. The MS is placed on the north east side of the crosssection at a distance of $3 \mathrm{~m}$ from the road and a height of 10 $\mathrm{m}$. The size of the MS is $50 \times 50$ controller elements with a suitable controller interspacing to account for the operating frequency of $26 \mathrm{GHz}$. We consider two scenarios: a) The case of the reflected signal targeting a single vehicle, and b) the case of the reflected signal targeting 2 vehicles on different lanes thus moving in opposite directions. The angular step is chosen to be $5^{\circ}$. To characterize the spatio-temporal workload incurred in each of the scenarios we use heat maps depicting the frequency of reconfiguration of each of the controllers within the MS. The deeper the color the larger the number of times the specific controller needs to reconfigure throughout the simulation experiment.

The heat maps for the single and multi-user case are shown schematically in Fig. 2(a) and Fig. 2(b) respectively with the latter corresponding to the case of the MS segmentation done via row sectoring. In the single user case there is a relatively even distribution of the load throughout the MS while in the multi-user case, the lower half of the MS bears most of the load as result of the row sectoring approach. If column sectoring is applied the right half region bears most of the load. The two cases are also compared in terms of the total number of messages disseminated. 17467 reconfiguration packets are disseminated in the case of a single user case while the corresponding number for the 2 user case is 24612. This demonstrates that tracking 2 users does not incur double the load strengthening the benefits of the multi-user functionality.

\section{WORKLOAD REDUCTION}

In this section, we demonstrate further how the specifics of the receiver movement in vehicular scenarios within city streets which is in many cases linear can assist in significantly reducing the number of messages which have to be sent within the MS in order to realize vehicle tracking along its trajectory.

To exemplify the reasoning we consider a MS of size $50 \times 50$ tracking a linear target with an average velocity $50 \mathrm{~km} / \mathrm{h}$. To identify the nodes that mostly obtain the same state throughout the time of the considered motion, we generate clusters of controller nodes which report a high probability $p>0.7$ of obtaining the same state. Such clusters are depicted graphically in Fig. 3(a) with the nodes belonging to such a cluster having the same color. It is to be noted that a large portion of the nodes have a high probability of having the same state and thus the small number of color variations in the color 


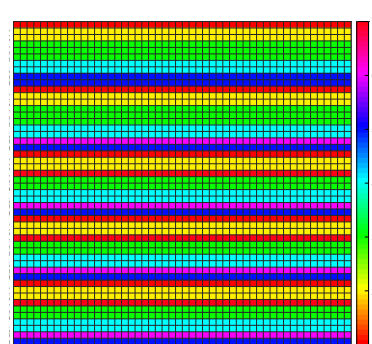

(a)

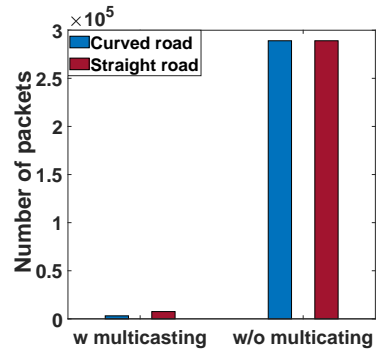

(b)
Fig. 3: (a)Spatial correlation in cell states. (b)Reduction in workload when multicasting is employed

scale. Furthermore, the states are distributed on the rows of the network such that cells on the same row have a high probability of acquiring the same state. This is different than what is observed in other type of movements which are not shown here due to lack of space. These results motivate the possible use of multi-cast routing in the controller network besides uni-casting which is currently considered for routing.

To evaluate the degree to which the communication burden may be reduced through multi-casting, we consider the following scenario. We consider a straight line road of length $1 \mathrm{~km}$ and vehicles utilizing the road with an average speed of $20 \mathrm{~km} / \mathrm{h}$. The vehicle traces are once again generated on VISSIM and the injection rate within the road is set to 50 $v e h / h$. In addition, to consider the case of multiple users being served using time division multiplexing of the MS resources we switch between two users. The two users are chosen $70 \mathrm{~m}$ apart at the initial stage of the simulation. We consider two scenarios. Scenario A at which one by one routing is employed to reconfigure each meta-atom and Scenario B where along a particular row the reconfiguration of the far end meta atom is used as a means to reconfigure all meta-atoms along the row as the packet traverses from source to destination. This can be achieved by means of adding a single bit in the packet header to indicate multi-casting where a variant of the XY-algorithm is employed which reconfigures all nodes sharing the same $\mathrm{Y}$ coordinate with the destination.

The same experiment is repeated when the roadway is slightly curved. The results, depicted in Fig. 3(b), report a significant reduction in the number of messages sent.

\section{UTILITY vs WORKLOAD OPTIMIZATION}

In this section, we explore how the workload within the MS can affect protocol design for IRS assisted communications in vehicular environment. We assume slow moving vehicles, pedestrians or cyclists which all claim mmwave access to the blocked vehicle via the MS. Due to their slow moving nature, it is highly likely that once they are served by targeting the reflected beam towards them they stay within the beam width before another user is served. This ensures that no switching is necessary for the purpose of maintaining the target within the beamwidth, thus correlating the switching with the targeted receiver. The problem is to serve as many users as possible by switching between them. Switching too often satisfies the aforementioned objective at the expense, however, of a larger workload incurred on the MS. This hints towards a multiobjective optimization problem where the objective is to maximize the aggregate utility and at the same time minimize the workload. As these two objectives are conflicting, an aggregate function must be considered. We consider a maxmin problem where the objective is to maximize the minimum of the cost functions once they are appropriately scaled. The resulting optimization problem is solved to yield an optimal switching frequency of $300 \mathrm{~ms}$ which is fairly unaffected by other factors such as the MS size. Below we provide details as to the adopted procedure.

The considered scenario involves dividing a straight line road into sections of length $L$ and at each time instant a single user is served in that section. The MS reconfigures every switching period $T$, changing the reflection angle of the incident wave so that a vehicle from an adjacent section is served. The switching sequence involves a cyclic policy, serving sections from left to right sequentially and once the rightmost has been reached, the sequence restarts. To formulate the optimization problem we need to characterize the capacity of the transmitter to receiver channel via the MS which in turn implies that the received power must be characterized as a function of the transmitted power. Different models have been proposed and evaluated in recent literature [16] assuming that each unit cell on the MS can incur an arbitrary phase/amplitude shift. In this work, we adopt a simpler approach, taking advantage of the fact that the MS coding procedure provides a characterization of the radiation pattern of the reflected beam. As the transmitter is assumed fixed, we assume that the power reflected from the MS is also fixed and that the received power is characterized by the MS-receiver channel, with the radiation pattern of the reflected beam obtained from the MS coding procedure. A well established data rate model in vehicular settings is the one described in [7] which relies on the assumption that the receive gain is fully characterized by the beamwidth (assuming negligible sidelobes). In our case, the beamwidth of the reflected beam can be readily obtained from the radiation pattern [14]. According to [7], the received power can be expressed as:

$$
P_{r x}(t, B W)=K\left(\frac{\pi^{2}}{B W^{2}}\right)^{2} \frac{1}{\left(r(t)^{2}\right)^{\frac{n}{2}}}
$$

where $r(t)$ is the distance between the receiver vehicle and the MS which is changing with time as the vehicle is moving, $B W$ is the beamwidth, $n$ is the pathloss exponent and $K$ is defined in [7] with the bandwidth set to $500 \mathrm{~Hz}$ with an operating frequency of $26 \mathrm{GHz}$ yielding the data rate $R(t, B W)$.

The utility of user $i$ is expressed as follows:

$$
U_{i}=\log (R(t, B W) * T)
$$

where $T$ is the switching period. Over the simulation time $L$, the total number of vehicles served are denoted by $N$ and thus the aggregate utility of all vehicles is calculated as $U=\sum_{i=1}^{N} U_{i}$. The choice of logarithmic utility functions is common in the literature to account for the function that a 


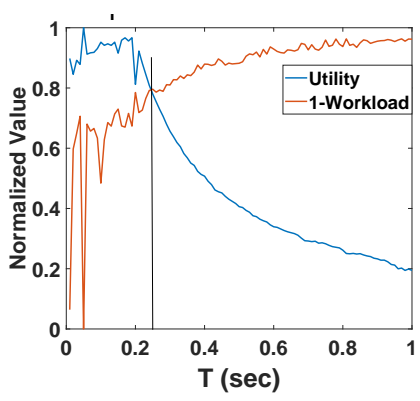

(a) Size $20 \times 20$

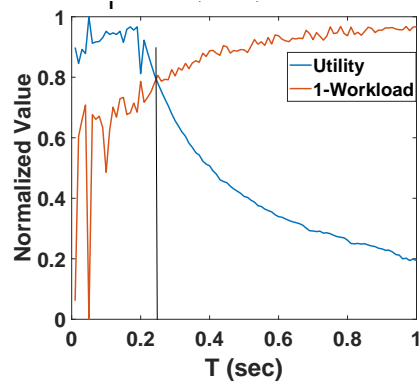

(b) Size $50 \times 50$
Fig. 4: Utility vs. the inverse of workload for different sizes and beam widths of the HyperSurface.

user is more "happy" if he is assigned a higher data rate, but an increase in sending rate allocation has a higher positive effect if this increase occurs at lower sending rates. The latter intuitive argument points towards concave utility functions with the logarithmic function constituting a popular choice. The multiplication by the period $T$ is done to transform the data rate into the actual data that the user receives. The longer a user is assigned the link, the more benefit he gets.

As the switching frequency decreases, the switching period increases with the total utility decreasing as less vehicles are served. To render the multi-objective optimization problem meaningful, the total utility is normalized by dividing by the maximum value. This guarantees that the normalized function attains values between 0 and 1 . The workload is negated to reflect the fact that the reward function increases as the switching frequency decreases since switchings are done less frequently. Normalization by appropriate scaling is also performed to bring the workload reward function attaining values between 0 and 1 . The normalized utility and workload functions $\tilde{U}(T), \tilde{W}(T)$ are then used to solve the following optimization problem:

$$
P: \max _{T} \min (\tilde{U}(T), \tilde{W}(T))
$$

The simulation scenario involves vehicle traces obtained from VISSIM on slow moving vehicles randomly distributed in the roadway. The results are depicted in Fig. 4. As expected, as the sampling frequency decreases (sampling period increases), the workload "utility" increases as the actual traffic decreases with less frequent reconfigurations. At the same time, the total utility decreases as less vehicles are served. The optimal sampling period where the two graphs intersect according to the optimization problem $P$ is at $300 \mathrm{~ms}$. It must be noted that the same experiment is repeated for different MS sizes with the results exhibiting similar behavior. Larger MSs are expected to incur larger traffic within the embedded controller network as more controller nodes need to be updated every reconfiguration. However, this workload increase comes at the benefit of a better electromagnetic performance in terms of the radiation pattern of the reflected beam being more focused as indicated by the smaller beam width.

\section{CONCLUSIONS AND Future WORK}

In this work we demonstrated the procedure with which the workload within the embedded controller network of a Hypersurface can be characterized for the case of multiple users, and we showcased the methodology in a vehicular scenario where the MS is used to alleviate the blockage problem on an intersection. Beyond that, we showed use cases of the workload characterization for information dissemination design within the embedded controller network and energy efficient communication protocol design for MS assisted communications in vehicular networks. Future research directions include per hop characterization of the workload towards a better description of the consumed power and formulation of realistic optimization problems aiming to minimize power consumption while at the same time optimize communication specific metrics in the case of multiple users.

\section{REFERENCES}

[1] K. Sakaguchi et al. Towards mmwave v2x in 5g and beyond to support automated driving. IEICE Transactions on Communications, 2020.

[2] T. Nitsche et al. Steering with eyes closed: Mm-wave beam steering without in-band measurement. In INFOCOM'15, page 2416-2424, 2015.

[3] S. Wang, J. Huang, and X. Zhang. Demystifying millimeter-wave v2x: Towards robust and efficient directional connectivity under high mobility. In Proceedings of MobiCom '20. ACM, 2020.

[4] M. A. ElMossallamy et al. Reconfigurable intelligent surfaces for wireless communications: Principles, challenges, and opportunities. IEEE Transactions on Cognitive Communications and Networking, 6(3):9901002, 2020.

[5] M. Di Renzo et al. Smart radio environments empowered by reconfigurable intelligent surfaces: How it works, state of research, and the road ahead. IEEE Journal on Selected Areas in Communications, 38(11):2450-2525, 2020.

[6] S. Gong et al. Toward smart wireless communications via intelligent reflecting surfaces: A contemporary survey. IEEE Communications Surveys Tutorials, 22(4):2283-2314, 2020.

[7] M. Ojaroudi, V. Loscri, and A. Vegni. Design and analysis of a reconfigurable intelligent meta-surface for vehicular networks. ITU Journal on Future and Evolving Technologies, 2020.

[8] Q. Wu et al. Intelligent reflecting surface aided wireless communications: A tutorial. IEEE Transactions on Communications, 2021.

[9] C. Liaskos, A. Tsioliaridou, S. Nie, et al. On the network-layer modeling and configuration of programmable wireless environments. IEEE/ACM Trans. Netw., 27(4):1696-1713, August 2019.

[10] C. Liaskos et al. A new wireless communication paradigm through software-controlled metasurfaces. IEEE Communications Magazine, 56(9):162-169, 2018.

[11] T. Saeed et al. Workload characterization of programmable metasurface. ACM NANOCOM'19, 2019.

[12] PTV Traffic Mobility Logistics. Accessed on April, 2021. [Online] Available: http://www.ptvamerica.com/vissim.htm.

[13] C. Huang et al. Reconfigurable intelligent surfaces for energy efficiency in wireless communication. IEEE Transactions on Wireless Communications, 18(8):4157-4170, 2019.

[14] H. Taghvaee et al. Scalability analysis of programmable metasurfaces for beam steering. IEEE Access, 8:105320-105334, 2020.

[15] H. Taghvaee et al. Error analysis of programmable metasurfaces for beam steering. IEEE Journal on Emerging and Selected Topics in Circuits and Systems, 10(1):62-74, 2020.

[16] W. Tang et al. Wireless communications with reconfigurable intelligent surface: Path loss modeling and experimental measurement. IEEE Transactions on Wireless Communications, 2020. 